\title{
STICKLER SYNDROME: ANAESTHETIC CONSIDERATIONS - A CASE REPORT.
}

Dr. Ibemhal Heisnam, Dr. S .Thoibahenba Singh, Dr. K. Upendra Singh, Dr. P. Pritam Singh,

Dr. L. Chandramani Singh

1. Assistant Professor, Department of Anaesthesiology, Jawaharlal Nehru Institute of Medical Sciences. Imphal.

2. Assistant Professor, Department of Anaesthesiology, Jawaharlal Nehru Institute of Medical Sciences. Imphal.

3. Assistant Professor, Department of Anaesthesiology, Jawaharlal Nehru Institute of Medical Sciences. Imphal.

4. Senior Resident, Department of Anaesthesiology, Jawaharlal Nehru Institute of Medical Sciences. Imphal.

5. Senior Resident, Department of Anaesthesiology, Jawaharlal Nehru Institute of Medical Sciences. Imphal.

\section{CORRESPONDING AUTHOR}

Dr. Ibemhal Heisnam

Thangmeiband Khoyathong Polem Leikai,

Imphal - 795001.

E-mail: dribemhal@gmail.com,

Ph: 00919856153557.

ABSTRACT: BACKGROUND: Stickler Syndrome is a multi systemic disorder caused by genetic malfunction in the tissue that connects bones, heart, eyes and ears. Symptoms include myopia, cataract and retinal detachment, hearing loss, midfacial underdevelopment and cleft palate and mild spondyloepiphyseal dysplasia and or arthritis. This case reports discusses the systemic problems and anaesthetic management of a patient with stickler syndrome.

KEYWORDS: Stickler Syndrome, Hereditary arthro- opthalmopathy, difficult airway, inhalational induction.

INTRODUCTION: Stickler syndrome is a genetically linked multisystem connective tissue disorder. Its prevalence is estimated to be about 1 in 10000 population. It is inherited in an autosomal dominant manner and involves the tissue that connects bones, heart, eyes, and ears. Because of its inherent problems including airway management and multisystem involvement, safe anaesthetic management of such a case need careful planning and conduct of the same.

CASE REPORT: We present a case of Stickler syndrome in a 13 year old child presented with giant retinal tear at our hospital. Pars Plana Vitrectomy with sub retinal fluid drainage with silicon oil injection was done under general anaesthesia. Her complaint was sudden onset painless loss of vision of the right eye for the last 15 days. She was diagnosed to have retinal detachment of both the eyes, right more than the left and was also myopic with a power of $-7 \mathrm{D}$ in the right eye and $-2 \mathrm{D}$ in the left eye. She weighs $40 \mathrm{kgs}$, her pulse rate was $80 / \mathrm{min}$, B.P. was $110 / 70 \mathrm{mmHg}$ andSpO $\mathrm{O}_{2}$ was $98 \%$ in room air .On physical examination she had cleft palate (Fig. I), flat nasal bridge with a small nose (Fig. II), and micrognathia (Fig. III). The fingers and toes of both upper and lower limbs showed abnormal length (Fig. IV). She complained of chronic pain in both knee and ankle joint. There was no evidence of kyphoscoliosis. She had hearing defect and audiometry revealed sensory neural deafness of both ears. Clinical evaluation of cardiovascular and respiratory system was unremarkable and her IQ was normal .There was no other similar family history. All the routine investigation was done including an echocardiography, which was normal. X-ray of the spine was normal. On airway assessment, 
mouth opening was adequate; thyromental distance was around $5 \mathrm{~cm}$ and Mallampati grade II with cleft palate. Neck movement and spine was normal.

Patient was premedicated with $5 \mathrm{mg}$ oral diazepam on the previous night. On arrival in the operation theatre, pulse, B. P., ECG, and $\mathrm{SpO}_{2}$ were monitored. A trolley for difficult airway was kept ready in the theatre .The patient was premedicated with intravenous ondansetron 3 $\mathrm{mg}$ and $0.1 \mathrm{mg}$ glycopyrrolate. A gradual gas induction with sevoflurane was done after preoxygenating the patient for 3 mins. At a deeper plane laryngoscopic assessment was done which showed a Cormack and Lehane of glottis visualization as class II. Anaesthesia was deepened with propofol $2 \mathrm{mg} / \mathrm{kg}$ and fentanyl $2 \mu \mathrm{g} / \mathrm{kg}$ IV. After confirming adequate manual ventilation, succinylcholine $1.5 \mathrm{mg} / \mathrm{kg}$ was given and after a smooth larngoscospy patient was intubated with $6.5 \mathrm{~mm}$ I.D. endotracheal tube with the help of a bougie. Correct placement of the tube was confirmed by auscultation and appearance of $\mathrm{ETCO}_{2}$ in the monitor. Anaesthesia was maintained with nitrous oxide in oxygen mixture and sevoflurane and vecuronium $(0.08 \mu \mathrm{g} / \mathrm{kg})$ and fentanyl $(0.5 \mu \mathrm{g} / \mathrm{kg})$. The vitals were stable throughout the surgery which lasted for about two hours. The patient was reversed with IV neostigmine and glycopyrrolate at the end of the surgery and extubation was done once the child was fully awake. Her postoperative recovery was uneventful and she was discharged after three days.

DISCUSSION: Stickler Syndrome also known as 'Hereditary arthro-opthalmopathy' is a connective tissue disorder first described by Dr. Gunnar Stickler in 1960. It is a multi system disorder that can affect the eyes and ears, skeleton and joints and craniofacies. Symptoms may include myopia, cataract and retinal detachment, hearing loss that is both conductive and sensorineural, mid facial underdevelopment and cleft palate, mild spondyloepiphyseal dysplasia and/or precocious arthritis. This case report is an attempt to discuss the characteristic features of Stickler Syndrome and possible problems which can be encountered during anaesthesia.

Stickler Syndrome arises due to mutation of gene involved in collagen synthesis and is inherited in an autosomal dominant manner. But in few cases spontaneous mutation may occur meaning it occurs with no prior family history as in our patient. The diagnosis of Stickler Syndrome is clinically based. The diagnosis can be confirmed by molecular genetic testing, however, it is primarily used to obtain information for genetic counseling. The disorder should be considered in individuals with clinical findings in two or more of the following categories. :

i) Ophthalmologic: Congenital or early onset cataract congenital vitreous anomaly, rhegmatogenous retinal detachment, myopia $>-3$ Dioptres

ii) Cranio facial: Midface hypoplasia producing a flat facial profile referred as scooped out face, bifid uvula, cleft palate, micrognathia, Robin sequence( micrognathia, cleft palate, glossoptossis)

iii) Audiologic: Sensorineural or conductive hearing loss, hyper mobile middle ear system

iv) Joints: Hypermobility, mild spondyloepiphyseal dysplasia, precocious osteoarthritis and spinal abnormalities

v) Cardiovascular system: mitral valve prolapse has been reported in almost $50 \%$ of the individuals with Stickler Syndrome. 
Peter S Rose et al in their study described in detail the clinical characteristics of Stickler Syndrome and proposed a diagnostic criteria ${ }^{1}$. Our patient was a classical case with almost all the features in their diagnostic criteria. Anaesthetic consideration includes possibility of difficult airway due to craniofacial abnormality and mitral valve prolapse causing cardiac problem. No cardiac abnormality was detected in our patient. Micrognathia ${ }^{2}$ and cleft palate may cause airway difficulties. But the characteristic facial appearance of Stickler Syndrome tends to improve with age, micrognathia tends to become less prominent over time, so anaesthetic problem tend to decrease in this 13 year old patient. The younger the patient, the more is the problem with airway management. ${ }^{3}$

A proper and careful airway assessment ${ }^{4}$ helped us to plan our anesthetic technique. Since our patient was a little older, her airway was not much distorted. But still we anticipated a difficult airway due to the presence of micrognathia and cleft palate, so necessary precaution was taken. Although, awake intubation is the gold standard for difficult intubation, we did not try it because of lack of coordination from the child. Retrograde intubation is very uncomfortable and traumatic to awake patients especially in paediatric age group. Laryngeal mask airway (LMA) ${ }^{5}$ is another good alternative in patient with difficult airway. Following insertion, it may be used to maintain the airway, or as a route to allow tracheal intubation. Parul Mullick ${ }^{6}$ et al have reported a case of Pierre- Robin syndrome in which LMA was used as a conduit for endotracheal intubation. Fiber optic endotracheal intubation through LMA in a child with Treacher Collins Syndrome has also been reported by Lisa Muraka ${ }^{7}$ et al.

Deep inhalational induction of anaesthesia ${ }^{8}$ is also a widely used technique for patient anticipated to be difficult to intubate. If airway obstruction develops, anaesthesia may be turned off and the patient woken up. During induction, when the patient is deeply anaesthetized, direct laryngoscopy is performed and if the larynx is visible, the patient may be intubated directly or be given a muscle relaxant and then intubated. In our patient, after deep inhalational induction, laryngoscopy showed a Cormack Lehane ${ }^{9}$ of glottis visualization as class II and manual ventilation was adequate so we decided to give muscle relaxant and intubate the patient smoothly. Inhalational induction and intubation in deeper plane after directly assessing the airway in a child with Treacher Collins syndrome was reported by Leena Goel ${ }^{10}$ et al. Paul J Kuzma et al have also reported successful intubation after deep inhalational induction in a patient with multiple pterygium syndrome. ${ }^{11}$

Other problems of Stickler Syndrome includes early onset of osteoarthritis which may be severe, leading to surgical joint replacement ${ }^{12}$ in younger age group. Due to precocious osteoarthritis these patients need great care during positioning.

CONCLUSION: The symptoms and severity of Stickler Syndrome vary from patient to patient even within the family. So anaesthetic technique should be individualized for every patient. Care should be taken when planning anaesthesia because airway management is often difficult in younger age group and emergency surgery may be required. 


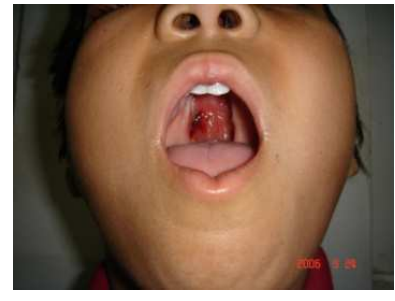

Fig .I: Cleft Palate with Receding Mandible

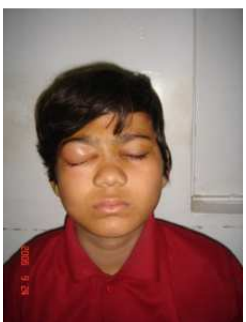

Fig. II: Front view showing Flat Nasal Bridge

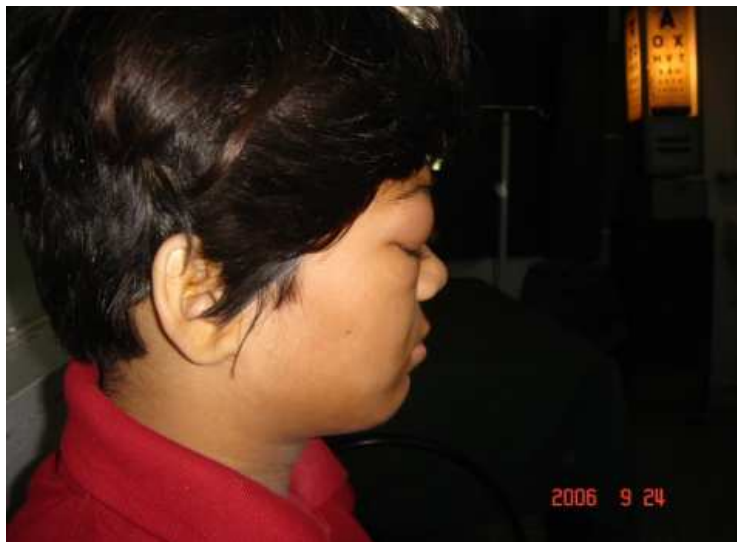

Fig. III: Lateral view showing Micrognathia
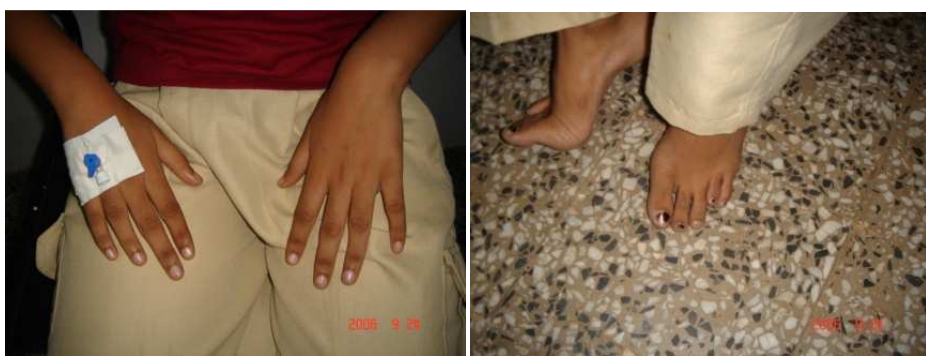

Fig. IV: Skeletal Abnormalities of both Hands and Feet

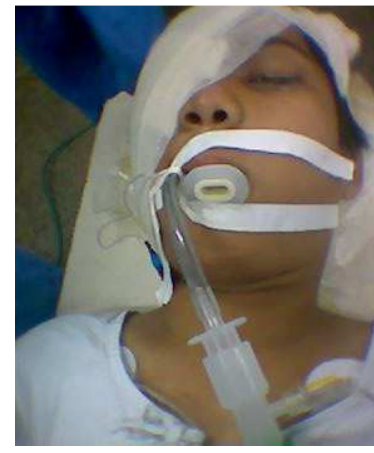

Fig V: Patient under Anaesthesia 


\section{REFERENCES:}

1. Peter S. Rose, Howard P. Levy, Ruth M. Liberfarb, Joie Davis, Y.Szymko-Benett, Benjamin I. Rubin et al. Stickler Syndrome: Clinical characteristics and diagnostics criteria. American Journal of Medical Genetics (2005) 138 A: 199- 207

2. Kücükyavuz Z, Ozkaynak 0 , Tüzüner AM, Kisnisci R. Difficulties in Anesthetic management of patients with micrognathia: Report of a patient with Stickler Syndrome. Oral Surg Oral Med Oral Pathol Oral Radiol Endod. 2006 Dec; 102(6): e33-6

3. Yokota Hiroshi, Takahata Osamu, Sengoku Kazufumi, Okada Hanako, Iwasaki Hiroshi. Anesthetic management of a patient with Stickler Syndrome. Masui (2006) Vol 55; No. 12; Page 1487- 1489

4. Dr Sunanda Gupta, Dr Rajesh Sharma KR, Dr Dimpel Jain. Airway Assessment: Predictors of Difficult Airway. Indian J. Anaesth. 2005; 49(4): 257-262

5. Peery, Michael. Airway Management of the Severely Retrognathic Child: Use of the Laryngeal Mask Airway. Ear Nose and Throat Journal 2002/April/1-p583, ISSN 01455613

6. Dr. Parul Mullick, Dr Vandana Talwar, Dr Anoop Raj Gogia. The laryngeal mask- an aid for difficult intubation in a child with Pierre - Robin Syndrome- A case report. Indian J. Anaesth. 2005; 49(1): 51-53

7. Lisa Muraika, Julius S. Heyman, Yuri Shevchenko. Fibreoptic tracheal Intubation through a laryngeal mask airway in a child with Treacher Collins Syndrome. Anesth Analg 2003; 97;1298-9

8. Dr I H Wilson, Dr Andreas Kopf. Prediction and Management of Difficult Tracheal Intubation. Update in Anaesthesia Issue 9 (1998) Article 9

9. Cormack RS, Lehane J. Difficult Tracheal Intubation in Obstetrics. Anaesthesia 1984; 39: $1105-1111$

10. Leena Goel, Santosh Kumar Bennur, Shweta Jambhale. Treacher Collins Syndrome - A challenge for Anaesthesiologist. Indian J. Anaesth. 2009; 53(4): 496-500.

11. Paul J Kuzma, Mark D. Calkins, Mark D. Kline, Steven M. Karan, Michael D. Maston. The Anaesthetic Management of Patients with Multiple Pterygium Syndrome. Anaesth Analg 1996; 83: 430-2.

12. GopalKrishna G. Verma, Adel Zarough, KH Suraliwala. Surgical difficulties for total knee replacement in Stickler Syndrome: A case report. Cases Journal 2008, 1:179. 